\title{
ниох New adamantane derivatives containing monoterpene fragments in their structure and investigation of their antipox activity
}

\author{
E. Mozhaitsev ${ }^{1 *}$, E. Suslov ${ }^{1}$, D. Rastrepaeva ${ }^{1,3}$, D. Korchagina ${ }^{1}$, N. Bormotov², O. Yarovaya ${ }^{1,3}$, O. Serova ${ }^{2}$, A. Agafonov ${ }^{2}$, \\ R. Maksyutov², L. Shishkina ${ }^{2}$
}

1) N.N. Vorozhtsov Novosibirsk Institute of Organic Chemistry of Siberian Branch of Russian Academy of Sciences, Lavrentjev Avenue 9,630090 Novosibirsk, Russia. 2) Department of prevention and treatment of especially dangerous infections, State Research Center of Virology and Biotechnology VECTOR, Koltsovo, Novosibirsk Region, 630559, Russia

3) Novosibirsk State University, Pirogova St. 2, 630090 Novosibirsk, Russia

*mozh@nioch.nsc.ru

Since, WHO declared eradication of natural smallpox in 1980, Independent Advisory Group on Public Health Implications of Synthetic Biology Technology Related to Smallpox report to the WHO Director-General noted the need to continue developing new low molecular weight agents against variola virus because of a number of reasons, such as discounted vaccination, possibility of smallpox spreading from permafrost solid, bioterrorism, potential danger of other orthopoxviruses circulating in animal population [1]

Despite a number of adamantane derivatives is known to demonstrate antiviral activity, only few adamantane derivatives showed activity against vaccinia virus [2]. On the other hand, monoterpene derivatives demonstrate various biological activities, in particular some camphor derivatives were found being active against vaccinia virus [3]. To find new agents to be active against orthopoxviruses we synthesized a different adamantane derivatives containing monoterpene fragments in their structure $[4,5]$. For this purpose a wide number of monoterpene amines was obtained starting from corresponding alcohols or carbonyl compounds. Isocyanates, isothiocyanates containing 1- or 2- substituted adamantane fragment were obtained as well. Combination of carboxylic acids chlorides or isocyanates and isothiocyanates with amines led to a number of amides, ureas or thioureas combining adamantane and monoterpene fragments in their structure.

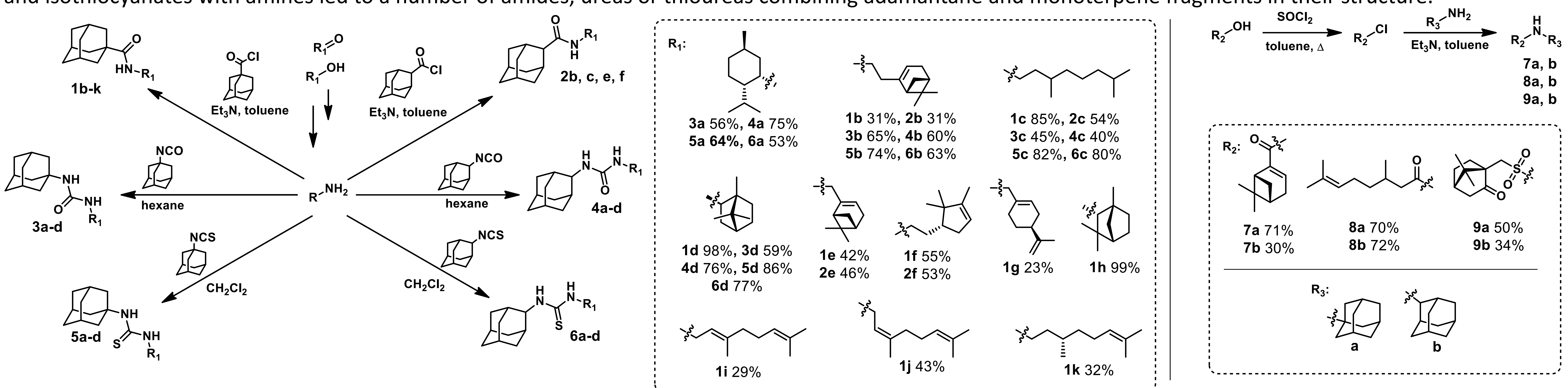

Amides $\mathbf{1 b}-\mathbf{k}, \mathbf{2} \mathbf{b}, \mathbf{c}, \mathbf{e}, \mathbf{f}, \mathbf{7} \mathbf{a}, \mathbf{b}, \mathbf{8} \mathbf{a}, \mathbf{b}, \mathbf{9} \mathbf{9}, \mathbf{b}$ were tested against vaccinia virus, it was shown that the derivatives of bicyclic (pinene and bornane) monoterpenes possess a most potent antipox activity. For derivatives $\mathbf{1 b}, \mathbf{1 e}, \mathbf{2} \mathbf{b}, \mathbf{2 e}, \mathbf{7 b}$ antiviral activity against cowpox virus and ectromelia virus was shown as well.

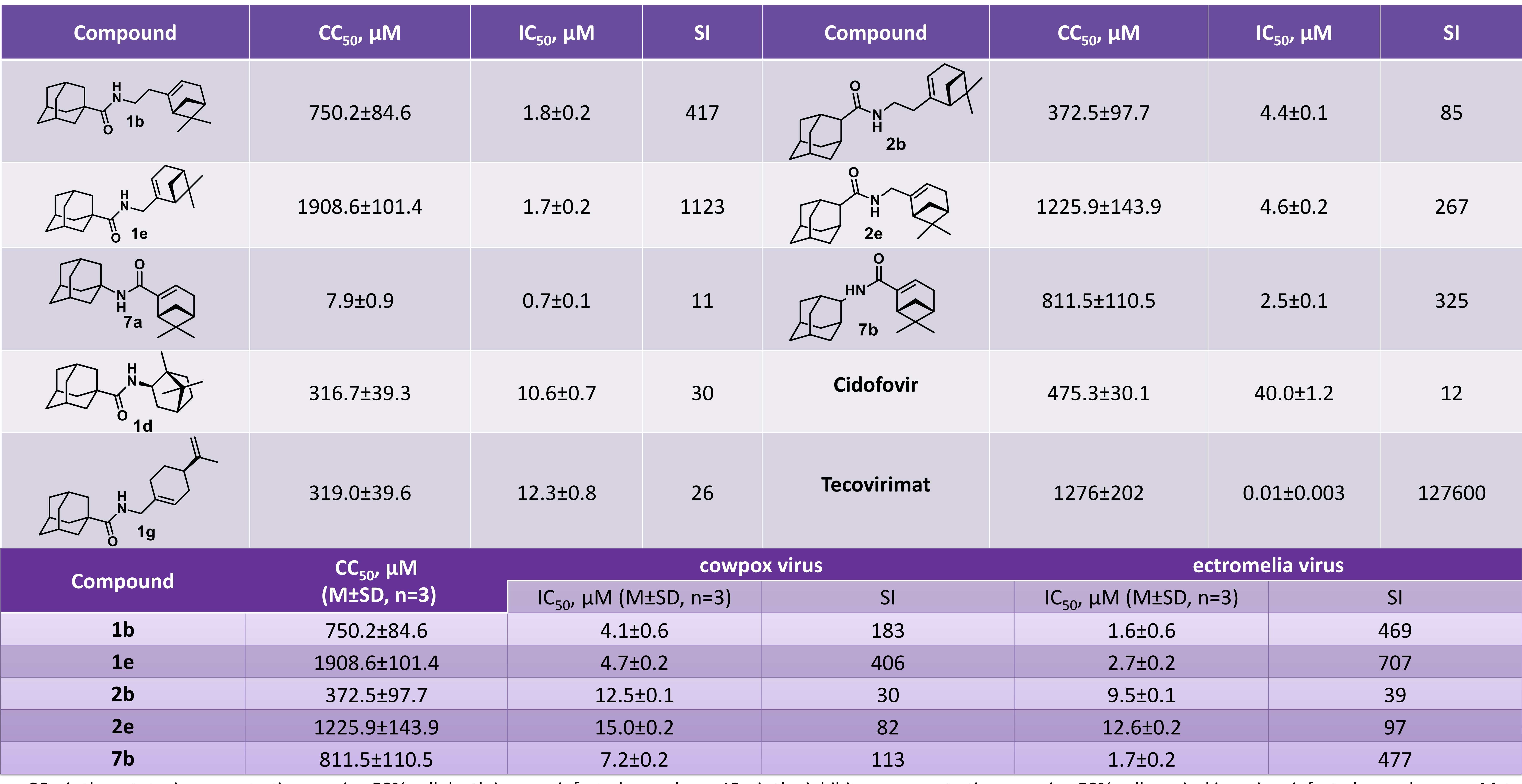

$\mathrm{CC}_{50}$ is the cytotoxic concentration causing $50 \%$ cell death in an uninfected monolayer; $\mathrm{IC}_{50}$ is the inhibitory concentration ensuring $50 \%$ cell survival in a virus-infected monolayer; as $\mathrm{M} \pm$ $S D$, where $M$ is the mean, and SD is the standard deviation; $n=3$ is the number of $C C_{50}$ and $I C_{50}$ measurements; $S I$ is the drug selectivity index $\left(C C_{50} / I C_{50}\right)$

References:

[1] World Health Organization

https://www.who.int/csr/resources/publications/smallpox/synthetic-biology-technologysmallpox/en/

[2] A. Kreutzberger et al., Arch. Pharm. (Weinheim, Ger.), 1984, 317, 767.
[3] A. Sokolova et al., Chem. Biodiversity, 2018, 15, 1.

[4] E. Suslov, et al. RSC Med. Chem. 2020, Doi. 10.1039/D0MD00108B

[5] A. A. Chepanova et al., Appl. Sci. 2019, 9, 1 